\title{
Role of Ambulatory Blood Pressure Monitoring in Predicting Adverse Maternofetal Events in Pregnancy
}

\author{
Satish Kumar Rao Vavilala ${ }^{1} \quad$ Indrani Garre ${ }^{1} \quad$ Sumalatha Beeram² \\ ${ }^{1}$ Department of Cardiology, NIMS, Hyderabad, India \\ 2OBG Gandhi Medical College, Secunderabad, India \\ Address for correspondence Dr. Satish Kumar Rao Vavilala, MD, DTCD, \\ Department of Cardiology, NIMS, Hyderabad, TS- 500 082, India \\ (e-mail: drsatishrao09@gmail.com).
}

Ind J Car Dis Wom 2021;6:17-24.

\begin{abstract}
Keywords

- Ambulatory Blood Pressure Monitoring (ABPM)

- hypertension

- pregnancy

- non-dippers

- antenatal

- postnatal complications
\end{abstract}

Aims To correlate the relationship between the ambulatory blood pressure parameters and the occurrence of the antenatal and postnatal adverse maternofetal events in pregnancy.

Methods Observational study designed for 50 pregnant patients who had an appointment to the obstetrics with abnormal blood pressure (BP) measurements and for whom ambulatory blood pressure monitoring (ABPM) was studied between January 2019 and June 2019. Data about age, personal history, obstetrics, family, body mass index (BMI), weight gain in pregnancy, values of blood pressure in the appointment, values recorded in ABPM, delivery and newborn, pregnancy and postpartum events, and follow-up of woman and child. Data were analyzed using descriptive and inferential statistics with Minitab 17.0 for Windows.

Results Patients demographic data, clinical history, and laboratory results, including the ABPM parameters, were compiled. Antenatal complications occurred in 22 patients (44\%), and postpartum complications were found in 41 patients (82\%) whose ABPM values were deranged. Antenatal complications were studied using the binary logistic regression analysis for calculating the role each factor played in the development of hypertension. In the sample studied, mean age was 24.980 with a standard deviation of 4.876 ( $p=0.003$; minimum age of 19 years and maximum age of 38 years), mean weight of patient was 63.71 with a standard deviation of $63.71(p=0.001)$, mean gravida was 1.780 with a standard deviation of $0.910(p=0.034)$, mean gestation weeks at presentation was 33.000 weeks with a standard deviation of $4.086(p=0.041)$, mean birth weight was 2.226 with a standard deviation of $0.797(p=0.000)$, mean maximum diastole was 109.22 with a standard deviation of $16.53(p=0.002)$, mean day maximum systole was 187.2 with a standard deviation of $203.5(p=0.009)$, mean day minimum diastole was 63.50 with a standard deviation of $12.99(p=0.013)$, all of which had statistical significance. It is found that the nighttime diastolic blood pressure (DBP) and daytime maximum systolic blood pressure (SBP) were the best predictors of adverse events. Among antenatal complications (ANC), the most common complication is intrauterine growth restriction (IUGR), noted in ( $n=19,86.36 \%)$ preterm delivery $(n=17,77.27 \%)$ among the 17 babies who were delivered preterm; 12 (70.5\%) needed neonatal intensive care unit (NICU) care of which 4 (25\%) babies died because of prematurity; intrauterine death (IUD) was noted in 7 (31.81\%) patients and eclampsia was seen in 5 (22.72\%). Nondippers proðle had a worse survival rate at follow-up until published online

April 13, 2021
DOI https://doi.org/

10.1055/s-0040-1708576.
(C2021. Women in Cardiology and Related Sciences.

This is an open access article published by Thieme under the terms of the Creative Commons Attribution-NonDerivative-NonCommercial-License, permitting copying and reproduction so long as the original work is given appropriate credit. Contents may not be used for commercial purposes, or adapted, remixed, transformed or built upon. (https://creativecommons.org/licenses/by-nc-nd/4.0/).

Thieme Medical and Scientific Publishers Pvt. Ltd. A-12, 2nd Floor, Sector 2, Noida-201301 UP, India 
delivery compared with those with a dipper proðle. Postnatal complications were seen in 41 patients; among them, 13 patients (31.7\%) had abnormal fundus examination, 15 patients $(36.58 \%$ ) required usage of antihypertensive beyond first postpartum, 9 patients $(21.95 \%)$ required blood transfusion for severe bleeding in the form of postpartum hemorrhage. Binary logistic regression for systolic dippers versus nondippers shows statistical significance in age $(p=0.023)$, weight $(p=0.038)$, and para $(p=0.045)$ (-Table 3). Binary logistic regression for diastolic dippers versus nondippers shows statistical significance in age $(p=0.039)$, weight $(p=0.020)$, birth weight $(p=0.010)$, maximum heart rate $(p=0.043)$, and ANC $(p=0.007)$ Adverse events occurred most commonly in nondippers. Systole nondippers is noted in ( $n=41,82 \%$ ). Dippers is noted in ( $n$ $=9,18 \%)$, Diastole nondippers is noted in ( $n=39,78 \%)$ Dippers is noted in $(n=11,22 \%)$. Conclusion ABPM recorded blood pressure is very precise. ABPM is the advised method for both diagnostic and therapeutic monitoring of hypertensive pregnancy diseases, mainly in situations like whitecoat hypertension, masked hypertension, nocturnal hypertension, and nondipping profile. In patients with high-risk pregnancy, elderly primigravida, and precious pregnancy, who have a high-risk of developing pregnancy-induced hypertension (PIH) and related complications, early use of ABPM predicts adverse maternofetal events, which when intervened at an earlier date can prevent antenatal and postnatal complications.

\section{Abstract Image}

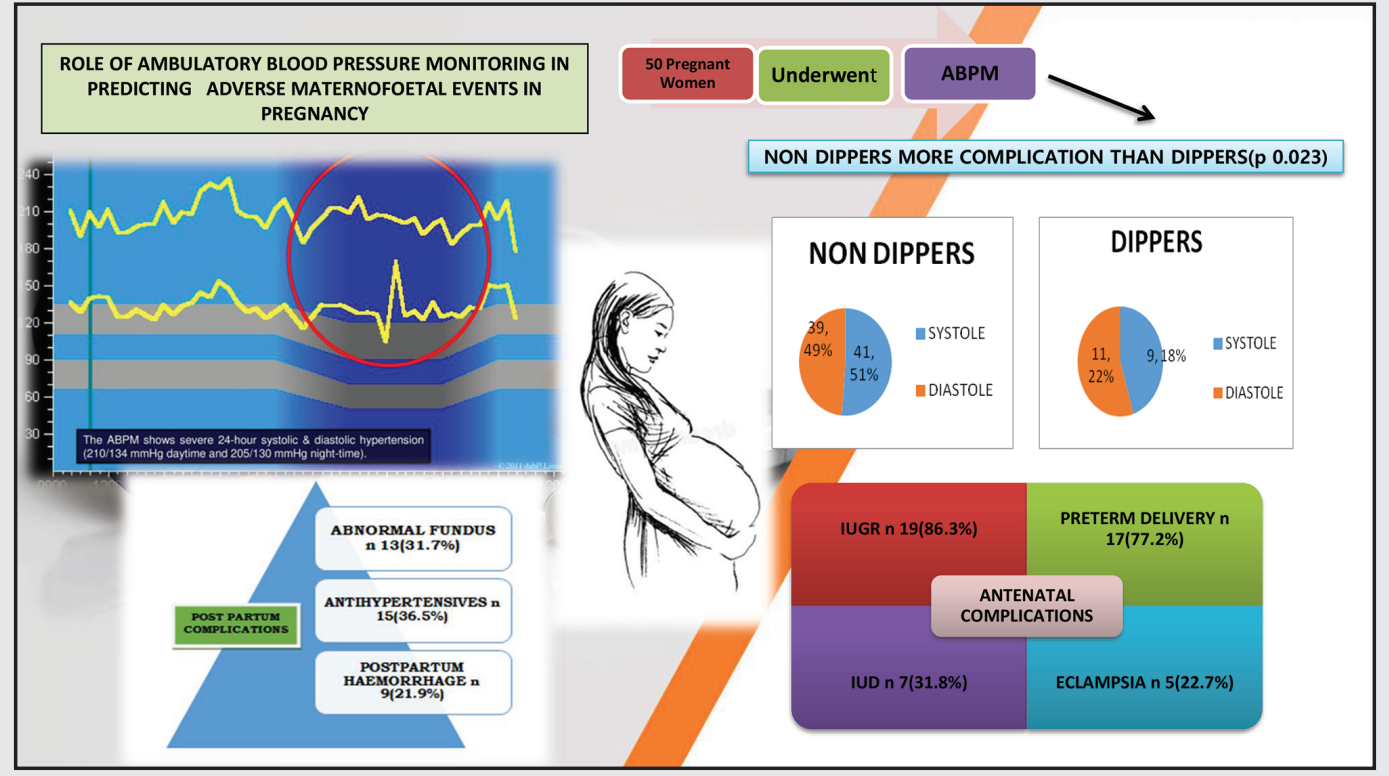

\section{Introduction}

Hypertension, being one of the commonly occurring diseases in the world, is emerging as the leading cause of premature morbidity and mortality globally. Office blood pressure (BP) readings will not reflect the patient's true $\mathrm{BP}$, with the possible limitations of false high or low BP recordings. Ambulatory blood pressure monitoring (ABPM), which has the additional benefit of detecting the dynamic variability of $\mathrm{BP}$, along with decreasing possible false recordings in the clinical settings, is the better predictor of major cardiovascular events. Absence of nocturnal dip in BP is an independent predictor of poor outcome.

Studies of ABPM have shown that cardiac complications of essential hypertension (left ventricular hypertrophy and stroke) are more frequently noted in patients whose 24-hour $\mathrm{BP}$ is flattened (nondippers); so, these patients are exposed to a longer duration of high $\mathrm{BP}$ throughout the day. If daytime period is taken as 0600 to 2200 hours and nighttime period is taken as $2200-0600$ hours, and the patients with hypertension whose nocturnal reduction of less than $10 \%$ in average 
daytime systolic blood pressure (SBP) and diastolic blood pressure (DBP) are classed as nondippers, the prevalence of these nondippers among patients with essential hypertension is around $35 \%$. Left ventricular mass is greater in nondippers than in dippers among women, but not in men. About 6 to $10 \%$ of pregnancies are complicated by pregnancy induced hypertension (PIH), and 1 to $5 \%$ of pregnancies ${ }^{1}$ are complicated by preexisting hypertension (HTN). The definition of hypertension in pregnancy is based on absolute BP values (SBP $\geq 140 \mathrm{~mm} \mathrm{Hg}$ or DBP $\geq 90 \mathrm{~mm} \mathrm{Hg}$ ), and distinguishes mildly (140-159/90-109 mm Hg) or severely ( $\geq 160 / 110 \mathrm{~mm}$ $\mathrm{Hg}$ ) elevated BP. For diagnosis, at least two recordings are taken at least 4 hours apart, but in severe cases, recordings are taken within minutes for confirmation. ${ }^{2}$ Conventional BP measures used for the diagnosis of HTN and the therapeutic efficacy of drugs.

BP measurements have some limitations when measured randomly, as it represents minimal circadian BP profile, effect of white coat, and technique which has potential failures.

Whitecoat hypertension, which is high-clinic BP but normal out-office BP in untreated and mixed patients, is linked with long-term adverse events of cardiovascular disease when compared with normotension people. ${ }^{3}$

ABPM is the method of recording blood pressure measurements for 24 hours and evaluating the circadian rhythm and various parameters like mean BP, pressure loads, areas under the curve, variations between daytime and night time, and pulse pressure variability. ${ }^{4}$ Nocturnal hypertension is defined as a BP more than $110 / 65 \mathrm{~mm} \mathrm{Hg}$ (lowered from the earlier value of $\geq 120 / 70 \mathrm{~mm} \mathrm{Hg}$ at night) as per ACC/AHA 2017 guidelines. ${ }^{5}$ From the Spanish ambulatory blood pressure monitoring registry, the prevalence of nocturnal HTN was more than $40 \%$ in the untreated, and $50 \%$ in the treated group. Nocturnal dipping is most commonly seen in females, but nocturnal HTN is more commonly seen in the male population. ${ }^{6}$

Therefore, 24-hour ambulatory monitoring is one of the best predictors of cardiovascular risk in the individual patient and nighttime, pulse pressure variability. Moreover, it is the only method for describing the circadian rhythm of blood pressure accurately, which is even more important when considering pregnant women. ${ }^{7}$

HTN is the most common complication seen during a complicated pregnancy, which can manifest as gestational HTN, preeclampsia, chronic HTN, or preeclampsia superimposed on chronic HTN. During pregnancy, women who are presenting with HTN-related complications have an increased risk of subsequent cardiovascular diseases later in life. ${ }^{8}$

$\mathrm{PIH}$ is one of the important causes of maternal, fetal, and neonatal mortality and morbidity, according to WHO reports. Women who present with PIH are at an increased risk of abruption placentae, cerebrovascular events, end-organ failure, and disseminated intravascular coagulation. Fetus of mother who present with PIH are at an increased risk of developing intrauterine growth restriction (IUGR), prematurity, and intrauterine death (IUD). ${ }^{9}$

HTN diagnosed by ABPM is better than office BP recordings in predicting adverse maternofetal events in pregnancy.

\section{Aims}

To correlate the relationship between the ambulatory BP parameters and the occurrence of the antenatal and postnatal adverse maternofetal events in pregnancy.

\section{Methods}

Observational studies designed for 50 pregnant patients, who have a regular antenatal checkup in the obstetrics department with abnormal BP measurements and were selected for ABPM, were enrolled for the study between January 2019 and June 2019. Data about age, demographic data, personal history, obstetrics history, family history, body mass index (BMI), weight gain in pregnancy, blood pressure recordings at every appointment, ABPM parameters, pregnancy details, antenatal, intrapartum and postpartum events, and postpartum follow-up of woman and child were collected.

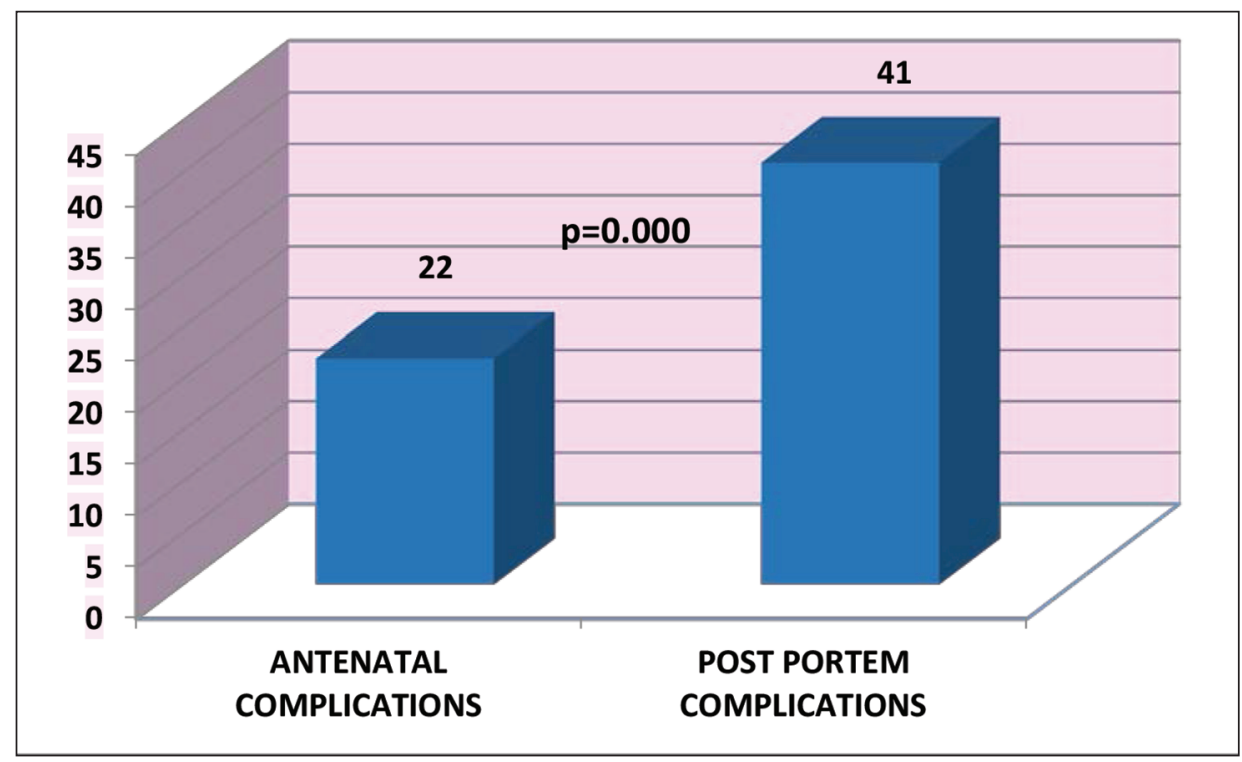

Fig. 1 Distribution of antenatal and postnatal complications. 
ABPM parameters are recorded by wearing on the nondominant arm a portable BP measuring device which is left for a 24 to 25 -hour period, so as to record the information pertaining to BP during day and night routine activities and sleep. The patient is asked to document the information regarding the symptoms and events that may alter BP and details regarding times of drug ingestion, food intake, and going to and getting up from bed.

Table 1 Descriptive statistics of study parameters

\begin{tabular}{|l|l|}
\hline Variable & Mean \pm St. Dev. \\
\hline Age $(\mathrm{yrs})$. & $24.98 \pm 4.87$ \\
\hline Height $(\mathrm{cm})$ & $160.6 \pm 43.2$ \\
\hline Weight $(\mathrm{kg})$ & $63.71 \pm 11.72$ \\
\hline BMl & $26.26 \pm 5.08$ \\
\hline Gravida & $1.78 \pm 0.91$ \\
\hline Present Gestation (weeks) & $33.00 \pm 4.086$ \\
\hline Birth Weight $(\mathrm{kgs})$ & $2.22 \pm 0.79$ \\
\hline Maximum systole $(\mathrm{mm} \mathrm{Hg})$ & $152.40 \pm 36.06$ \\
\hline Maximum diastole $(\mathrm{mm} \mathrm{Hg})$ & $109.22 \pm 16.53$ \\
\hline Day maximum systole $(\mathrm{mm} \mathrm{Hg})$ & $187.2 \pm 203.5$ \\
\hline Day maximum diastole $(\mathrm{mm} \mathrm{Hg})$ & $108.08 \pm 13.77$ \\
\hline Day minimum systole $(\mathrm{mm} \mathrm{Hg})$ & $106.38 \pm 13.64$ \\
\hline Day minimum diastole $(\mathrm{mm} \mathrm{Hg})$ & $63.50 \pm 12.99$ \\
\hline Abbrevitin: BMi body $\mathrm{mass} \mathrm{hex}$. & \\
\hline
\end{tabular}

Abbreviation: BMI, body mass index.

Table 2 Binary logistic regression for antenatal complications

\begin{tabular}{|l|l|}
\hline Variable & $p$-Value \\
\hline Age $(\mathrm{yrs})$. & 0.003 \\
\hline Weight $(\mathrm{kg})$ & 0.001 \\
\hline Gravida & 0.034 \\
\hline Present gestation (weeks) & 0.039 \\
\hline Birth weight $(\mathrm{kgs})$ & 0.000 \\
\hline Maximum diastole $(\mathrm{mm} \mathrm{Hg})$ & 0.002 \\
\hline Day maximum systole $(\mathrm{mm} \mathrm{Hg})$ & 0.009 \\
\hline Day minimum diastole $(\mathrm{mm} \mathrm{Hg})$ & 0.013 \\
\hline
\end{tabular}

In clinical practice, recordings are taken at 15 -minute intervals during the day and every 30 minutes overnight. The measurements are downloaded, so analyses of various parameters can be performed. Recordings are taken if, at least, $70 \%$ of daytime and night time recorded BP measurements are satisfactory, or else the test should be repeated.

Data was compiled, and analyzed using descriptive and inferential statistics with Minitab 17.0.

\section{Results}

Patients demographic data, clinical history, and laboratory results, including the ABPM parameters, were compiled. Antenatal complications occurred in 22 patients (44\%) postpartum complications found in 41 patients (82\%) whose ABPM values are deranged ( $\boldsymbol{- F i g .} \mathbf{1}$ ). Antenatal complication's studied using the binary logistic regression analysis for calculating the role each factor played in the development of hypertension in the sample studied mean age was 24.980 with a standard deviation of $4.876(p=0.003)$ (minimum age of 19years and maximum age of 38 years), mean weight of patient was 63.71 with a standard deviation of $63.71(p=$ 0.001 ), mean gravida was 1.780 with a standard deviation of

Table 3 binary logistic regression for systolic dippers versus nondippers

\begin{tabular}{|l|l|}
\hline Variable & $p$-Value \\
\hline Age (yrs.) & 0.023 \\
\hline Weight $(\mathrm{kg})$ & 0.038 \\
\hline Para & 0.045 \\
\hline
\end{tabular}

Table 4 Binary logistic regression for diastolic dippers versus nondippers

\begin{tabular}{|l|l|}
\hline Variable & $p$-Value \\
\hline Age (yrs.) & 0.039 \\
\hline Weight $(\mathrm{kg})$ & 0.020 \\
\hline Birth Weight (kgs) & 0.010 \\
\hline Maximum heart rate (bpm) & 0.043 \\
\hline Antenatal complications & 0.007 \\
\hline
\end{tabular}

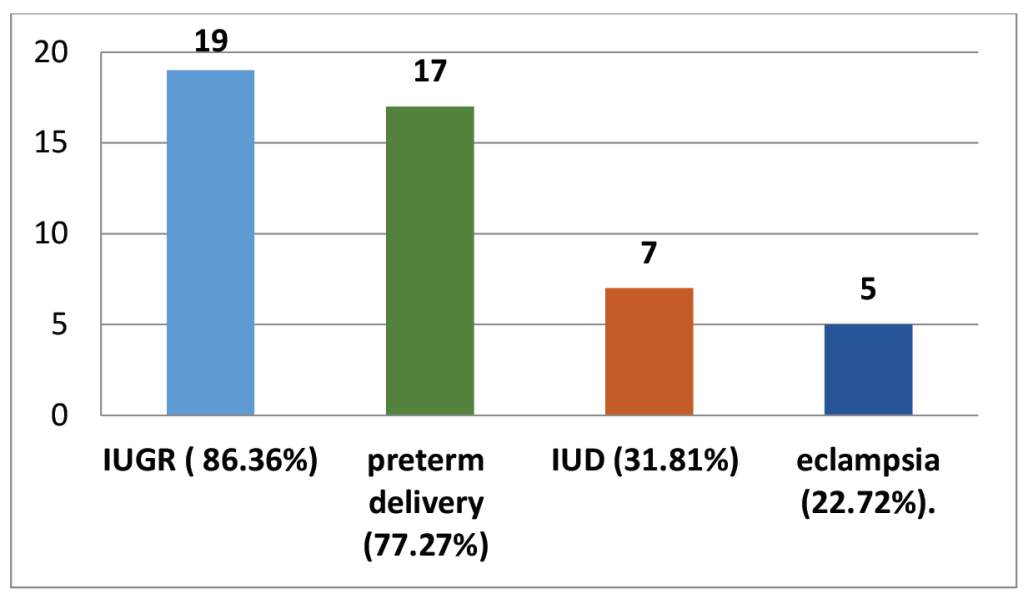

Fig. 2 Distribution of antenatal complications. 

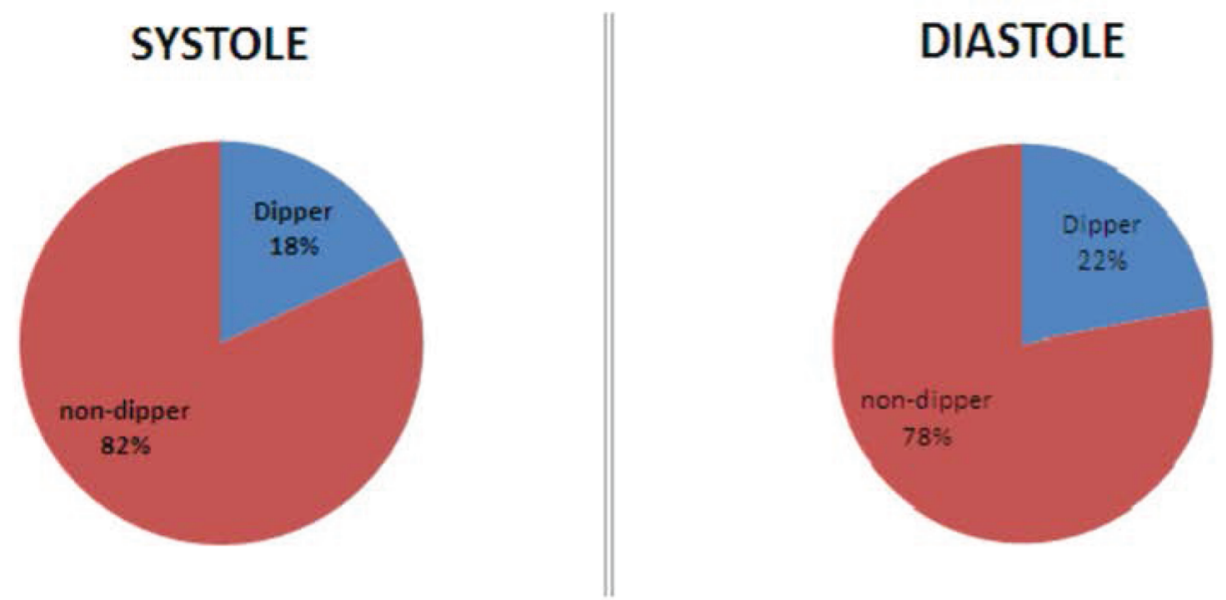

Fig. 3 Distribution of systole and diastole dippers and nondippers.

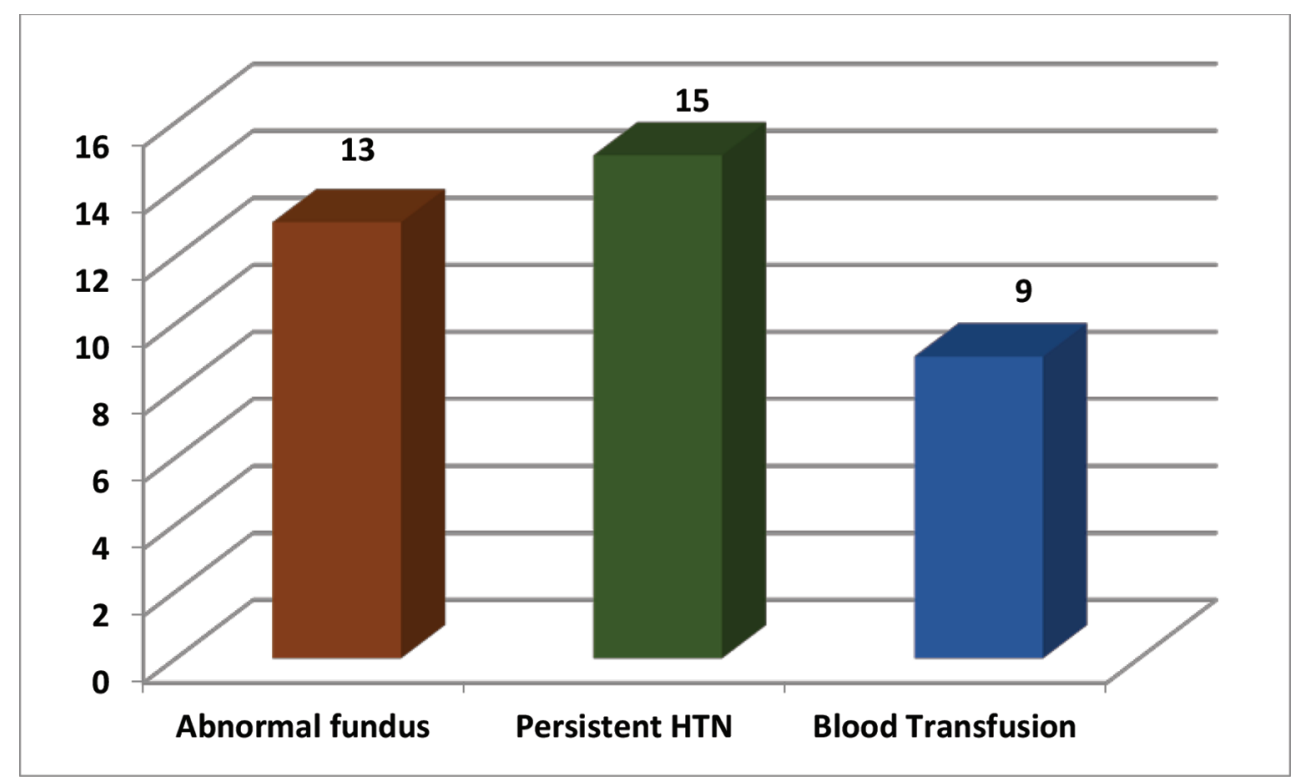

Fig. 4 Postpartum complications.

$0.910(p=0.034)$, mean gestation weeks at presentation was 33.000 weeks with a standard deviation of $4.086(p=0.041)$, mean birth weight was 2.226 with a standard deviation of $0.797(p=0.000)$, mean maximum diastole was 109.22 with a standard deviation of $16.53(p=0.002)$, mean day maximum systole was 187.2 with a standard deviation of 203.5 $(p=0.009)$, mean day minimum diastole was 63.50 with a standard deviation of 12.99 ( $p=0.013$ ) all of which had statistical significance $(-$ Table $\mathbf{1}, \mathbf{2})$. It is found that the night time DBP, day time maximum SBP were the best predictors of adverse maternofetal events. Among the antenatal complications (ANC), the most commonly noted is IUGR seen in $(n=$ $19,86.36 \%$ ) preterm delivery ( $n=17,77.27 \%$ ) among the 17 babies who were delivered preterm 12 (70.5\%) needed neonatal intensive care unit (NICU) care of which 4 (25\%) babies died because of prematurity, IUD was noted in 7 (31.81\%) patients, and eclampsia was seen in 5 (22.72\%) (-Fig. 2). Binary logistic regression for systolic dippers versus nondippers shows statistical significance in age $(p=0.023)$, weight $(p=0.038)$, and para $(p=0.045)$ (-Table 3$)$. Binary logistic regression for diastolic dippers versus nondippers shows statistical significance in age $(p=0.039)$, weight $(p=0.020)$, birth weight ( $p=0.010)$ maximum heart rate $(p=0.043)$, and ANC $(p=0.007)$ (-Table 4).

Adverse maternofetal events occurred most commonly in nondippers. Systole nondippers is noted in ( $n=41,82 \%)$ Dippers is noted in $(n=9,18 \%)$, Diastole nondippers is noted in $(n=39,78 \%)$ Dippers is noted in $(n=11,22 \%)$ (-Fig. 3 ). Nondippers profile had worse survival rate at follow-up until delivery compared with those with a dipper profile Postnatal complications were seen in 41 patients, of whom 13 patients (31.7\%) had abnormal fundus examination, 15 patients 


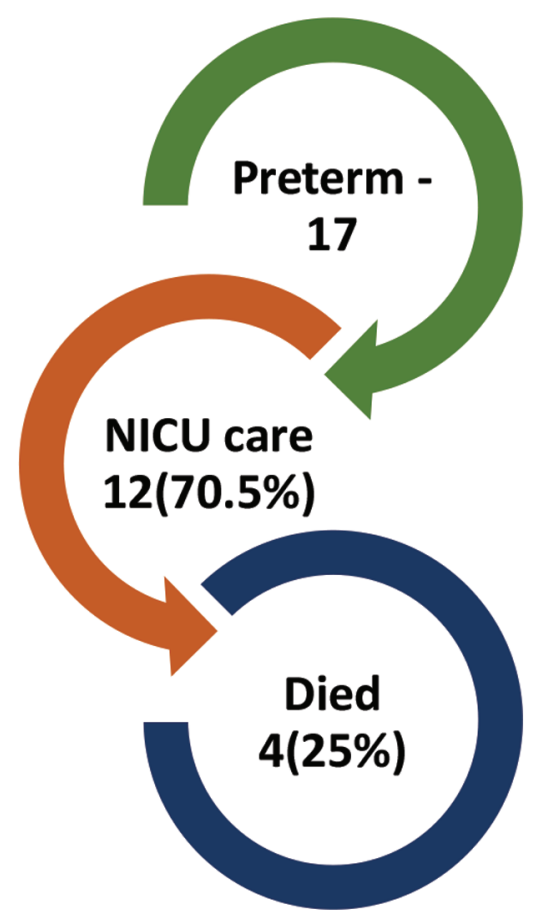

Fig. 5 Distribution of complications of preterm-born babies.

(36.58\%) required usage of antihypertensive beyond first postpartum, and 9 patients (21.95\%) required blood transfusion for severe bleeding in the form of postpartum hemorrhage (-Fig. 4).

\section{Discussion}

The main aim of our study is to demonstrate the value of ABPM in diagnosing and treating pregnancy-related HTN and also access few predictors of adverse maternal-fetal outcomes which, when addressed, can improve the outcomes. Twenty-four-hour SBPs and DBPs are not constant, which show characteristic circadian patterns seen in all individuals, including nonpregnant and pregnant women, in response to the internal clock and mental and physical activity.

ANCs occurred in 22 patients (44\%), and postpartum complications found in 41 patients (82\%) with abnormal ABPM (-Fig. 1). Women with mean maximum diastole, mean daytime maximum systole, and mean daytime minimum diastole had significant antenatal complications. Women with high nighttime DBP, that is, who do not show the physiological dipping of BP, along with daytime maximum SBP, are the best predictors for predicting adverse maternofetal events in pregnancy. Among ANCs, the most common noted complication is IUGR, which is seen in $(\mathrm{n}=19,86.36 \%)$ preterm delivery ( $\mathrm{n}=17,77.27 \%$ ), IUD was noted in 7 (31.81\%) patients, and eclampsia was seen in 5 (22.72\%) of women ( - Fig. 2). Among the 17 babies who were delivered preterm, 12 babies (70.5\%) needed NICU care, of which 4 (25\%) babies died because of prematurity and respiratory distress with low-Apgar scores (-Fig. 5). Adverse maternofetal events occurred most commonly in nondippers. Systole nondippers is noted in $(n=41$,
$82 \%)$. Dippers is noted in ( $n=9,18 \%)$. Diastole nondippers is noted in $(n=39,78 \%)$. Dippers is noted in $(n=11,22 \%)$ (-Fig. 3). Nondippers profile had worse events at follow-up until delivery compared with those with a dipper profile.

Postnatal complications were seen in 41 patients, of whom 13 patients (31.7\%) had abnormal fundus examination and were diagnosed with hypertensive retinopathy changes; 15 patients (36.58\%) required usage of antihypertensive beyond the first postpartum month, and 9 patients (21.95\%) required blood transfusion for severe bleeding in the form of postpartum hemorrhage ( - Fig. 4).

In 1962, the first published study was important in the evaluation of 24-hour ABPM without an observer, using a semi-automatic method. ABPM improves the diagnostic approach and the risk prediction in general hypertensive patients, and evidence also supports its usefulness in the management of hypertensive pregnancy disorders. ${ }^{10,11}$ Five decades ago, Kain et al showed the usefulness of ABPM and the ability to measure $\mathrm{BP}$ during a patient's routine activities. ${ }^{12} \mathrm{ABPM}$ is specifically advised for the identification of whitecoat HTN and masked HTN, as it shows an increase in daytime BP, nighttime BP, or both. Various studies showed that nocturnal BP might be relevant in pregnancy. ${ }^{13}$ In our study, we showed that females who show the absence of a physiological decrease in nocturnal BP (nondipper profile), especially the diastolic values, are important causes of adverse maternal-fetal events, which present superior and independent value in relation to the physiological decrease of BP values (dipper profile). According to the MEDLINE database in 2015, which scrutinized various articles from 2015, around 2000 studies showed the benefits of ABPM in confirming the diagnosis without false positive recordings, prognosis, and assessment of drug efficacy.

ABPM predicts the differing $\mathrm{BP}$ recordings throughout the gestation period for the healthy normotensive and hypertensive pregnant women. In normal pregnancy, BP gradually lowers till the middle of gestational period and then gradually increases till the day of delivery. In hypertensive pregnancy, with either gestational hypertension or preeclampsia, patients have constant BP during the first half of pregnancy, followed by a continuous linear BP increase till delivery. Studies have reported gender variations in the 24-hour ABPM and heart rate. Men show a lower heart rate and higher $\mathrm{BP}$ than women, with greater differences noted for SBP than DBP.

ABPM during pregnancy starting as early as first antenatal checkup after confirmed pregnancy is helpful in the early risk assessment, starting prophylactic or therapeutic medications, and can be taken as standard for the diagnosis of HTN in pregnancy.

ABPM is useful in monitoring BP among pregnant women, especially during the last trimester of pregnancy, without false recordings, by knowing the effect of the circadian rhythm on BP recordings. It can predict the outcomes of hypertensive patients with pregnancy, subject to increased risk of caesarean section. ${ }^{14}$ Normal values of ABPM in various trimesters of pregnancy are known, which are usually higher than conventional BP values. ${ }^{14}$ 
In our study, nocturnal HTN is the best predictor for the development of adverse maternal fetal events in pregnancy when it is adjusted for other potential confounders. The nocturnal diastolic values are the best predictors, which is statistically significant after adjustment of the other ABPM parameters. In the general population, nocturnal HTN is associated with end-organ damage and adverse cardiovascular events. It appears particularly relevant when considering nondipping circadian BP profiles are more commonly seen in preeclampsia.

White coat HTN, which accounts for approximately $30 \%$, can be detected by using ABPM according to a study carried out by Bellomo et al. Women diagnosed with white coat HTN can stop the use of antihypertensive without any adverse outcomes on pregnancy. ${ }^{15,16}$

The access of these values is only possible with BP monitoring methods like ABPM, but females with inadequate sleep duration or quality could selectively increase the nocturnal BP while, at the same time, affecting outcome. HTN in pregnancy, diagnosed by ABPM, is superior to the office measurements of BP in predicting outcomes and plays a role in predicting deterioration from gestational hypertension to adverse maternofetal events in pregnancy. ${ }^{17}$

\section{Limitations}

Small sample size, and ABPM parameters not compared with other markers of PIH.

\section{Conclusion}

ABPM-recorded BP is very precise. ABPM is the advised method for both diagnostic and therapeutic monitoring of hypertensive pregnancy diseases, mainly in situations like whitecoat HTN, masked HTN, nocturnal HTN, and nondipping profile.

ABPM in high-risk pregnant women can also help predict the occurrence of preeclampsia and/or IUGR, and other adverse maternofetal events which when intervened at an earlier date can prevent antenatal and postnatal complications; however, there are no guidelines which take into account its role in the management of these conditions, either antepartum or intrapartum.

\section{Future Prospects}

Nocturnal HTN is best associated with target organ damage and cardiovascular events. Nondipping circadian BP profiles are usually seen in patients with preeclampsia. These values are available with a method of blood pressure monitoring like ABPM.

With our study, we were able to improve clinical management in hypertensive disorders in pregnancy, emphasizing the importance of the nondippers of nocturnal DBP values, as predictors of adverse maternofetal events in the HTN-complicated pregnancies.
ABPM can become a useful tool in diagnosing HTN among pregnant woman, and it would also be possible to reduce false HTN and decrease adverse maternofetal events, which complicate pregnancy.

\section{Audio}

Audio file for this article is available at https://doi. org/10.1055/s-0040-1708576.

\section{Conflict of Interest}

None declared.

\section{References}

1 Regitz-Zagrosek V, Blomstrom Lundqvist C, Borghi C, et al; European Society of Gynecology (ESG)Association for European Paediatric Cardiology (AEPC)German Society for Gender Medicine (DGesGM)ESC Committee for Practice Guidelines. ESC Guidelines on the management of cardiovascular diseases during pregnancy: the Task Force on the Management of Cardiovascular Diseases during Pregnancy of the European Society of Cardiology (ESC) Eur Heart J 2011;32(24):3147-3197

2 American College of Obstetricians and GynecologistsTask Force on Hypertension in Pregnancy. Hypertension in pregnancy. Obstet Gynecol 2013;122(5):1122-1131

3 Huang Y, Huang W, Mai W, et al. White-coat hypertension is a risk factor for cardiovascular diseases and total mortality. J Hypertens 2017;35(4):677-688

4 Ogedegbe G, Pickering T. Principles and techniques of blood pressure measurement. Cardiol Clin 2010;28(4):571-586

5 Whelton PK, Carey RM, Aronow WS, et al. 2017 ACC/AHA/ AAPA/ABC/ACPM/AGS/APHA/ASH/ASPC/NMA/PCNA Guideline for the prevention, detection, evaluation, and management of high blood pressure in adults: executive summary: a report of the American College of Cardiology/American heart association task force on clinical practice guidelines. Hypertension 2018;71(6):1269-1324

6 Gijón-Conde T, Gorostidi M, Banegas JR, et al. [Position statement on ambulatory blood pressure monitoring (ABPM) by the Spanish Society of Hypertension (2019)]. Hipertens Riesgo Vasc 2019;36(4):199-212

7 Nobre F, Mion Junior D. Ambulatory blood pressure monitoring: five decades of more light and less shadows. Arq Bras Cardiol 2016;106(6):528-537

8 Gongora MC, Wenger NK. Cardiovascular complications of pregnancy. Int J Mol Sci 2015;16(10):23905-23928

9 Kintiraki E, Papakatsika S, Kotronis G, Goulis DG, Kotsis V. Pregnancy-induced hypertension. Hormones (Athens) 2015;14(2):211-223

10 Gupta HP, Singh RK, Singh U, Mehrotra S, Verma NS, Baranwal N. Circadian pattern of blood pressure in normal pregnancy and preeclampsia. J Obstet Gynaecol India 2011;61(4):413-417

11 Bilo G, Parati G. Ambulatory blood pressure monitoring: a mandatory approach in high-risk pregnancy? J Hypertens 2016;34(11):2140-2142

12 Kain HK, Hinman AT, Sokolow M. Arterial blood pressure measurements with a portable recorder in hypertensive patients. I. Variability and correlation with "casual" pressures. Circulation 1964;30:882-892 
13 Salazar MR, Espeche WG, Leiva Sisnieguez BC, et al. Significance of masked and nocturnal hypertension in normotensive women coursing a high-risk pregnancy. J Hypertens 2016;34(11):2248-2252

14 Brown MA. Is there a role for ambulatory blood pressure monitoring in pregnancy? Clin Exp Pharmacol Physiol 2014;41(1):16-21

15 Bellomo G, Narducci PL, Rondoni F, et al. Prognostic value of 24-hour blood pressure in pregnancy. JAMA 1999; 282(15):1447-1452
16 Brown MA. Is there a role for ambulatory blood pressure monitoring in pregnancy? Clin Exp Pharmacol Physiol 2014;41(1):16-21

17 Shahbazian N, Shahbazian H, Mohammadjafari R, Mousavi M. Ambulatory monitoring of blood pressure and pregnancy outcome in pregnant women with white coat hypertension in the third trimester of pregnancy. J Nephropharmacol 2013;2(1):5-9 